\title{
Las Podostemaceae como hábitat para los macroinvertebrados en arroyos de Misiones (Argentina)
}

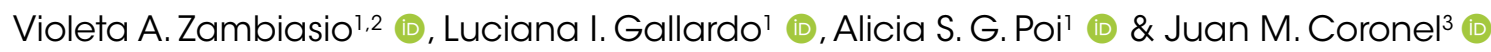

\begin{abstract}
1. Centro de Ecología Aplicada del Litoral - CECOAL (CCT Nordeste - CONICET- UNNE) Ruta 5, Km 2.5, (3400) Corrientes, Argentina. (guadalupepoi@gmail.com; lucianagallardo@hotmail.com)

2. Cátedra de Ecología y Medio Ambiente - FACENA, Universidad Nacional del Nordeste (UNNE), Corrientes, Argentina. (violetazambiasio@gmail.com)

3. Cátedra de Biología de los Invertebrados - FACENA, Universidad Nacional del Nordeste (UNNE), Corrientes, Argentina. (jm_coronel@yahoo.com)
\end{abstract}

Recibido 2 marzo 2019

Aceptado 27 junio 2019

Publicado 5 agosto 2019

DOI $10.1590 / 1678-4766 e 2019028$

\begin{abstract}
Podostemaceae as habitat for macroinvertebrates in streams of Misiones (Argentina). Podostemaceae are important components of the trophic networks of rheophilic environments but their importance as habitats for invertebrates is little known. In this study, the abundance and taxa richness of macroinvertebrate assemblages associated with Podostemum distichum and Podostemum muelleri (Malpighiales: Podostemaceae) in two low order streams were analyzed across seasons. Mean macroinvertebrate abundance differed significantly among seasons but did not differ between streams. Nine orders of macroinvertebrates were recorded in both streams, comprising 14 families. Non- metric multidimensional scaling (NMDS) reflected some differences in the seasonal pattern, too. The coefficient of similarity and the complementarity indicate that both streams were very similar in terms of the composition of the macroinvertebrate families. The dominant Podostemceae species in each stream constitute intermediate diversity habitats for macroinvertebrates according to the diversity index used.
\end{abstract}

KEYWORDS. Invertebrates, community structure, rheophilic environments.

RESUMEN. Las Podostemaceae son importantes componentes de las mallas tróficas de los ambientes reófilos pero su importancia como hábitat para los invertebrados es poco conocida. En este estudio se analizó la variación temporal de la abundancia y riqueza de taxa de los macroinvertebrados asociados a Podostemum distichum y Podostemum muelleri (Malpighiales: Podostemaceae) en dos arroyos de bajo orden durante diferentes estaciones del año. La abundancia total promedio difirió entre fechas de muestreo, pero no entre arroyos. Se registraron 14 familias de macroinvertebrados distribuidas en 9 órdenes en ambos arroyos. El Análisis de Escalamiento Multidimensional No Métrico (NMDS) reflejó también diferencias en el patrón estacional. Tanto el coeficiente de similitud como el de complementariedad, indican que ambos arroyos fueron muy similares en cuanto a la composición de las familias de macroinvertebrados. Las especies de Podostemaceae dominantes en cada arroyo constituyen hábitats de diversidad intermedia de acuerdo al índice de diversidad utilizado.

PALABRAS CLAVE. Invertebrados, estructura de la comunidad, ambientes reófilos.

En la Región Neotropical, la diversidad de plantas acuáticas es alta respecto de otras regiones (CHAMBERs et $a l ., 2008)$ y se debe, en gran parte, a la contribución de las podostemáceas (188 especies). En Argentina se encuentran seis géneros de Podostemaceae: Apinagia, Podostemum, Tristicha, Marathrum, Mourera y Wettsteiniola (TuR 1975, 1997, 1999, 2003) registrados en las cuencas de los ríos Paraná y Uruguay que rodean a la Mesopotamia Argentina. La distribución meridional de la familia en el río Paraná se extiende aproximadamente hasta la latitud $27^{\circ} \mathrm{S}$ y, en el río Uruguay, cerca de la latitud $31^{\circ} \mathrm{S}$. En los ambientes lóticos del Alto Paraná las Podostemaceae se encuentran sólo en las zonas de rápidos donde toleran velocidades de corriente de 2-3 $\mathrm{ms}^{-1}$ (NEIFF, 1986). Son escasos los trabajos existentes sobre la vegetación reófila de las provincias de Corrientes y Misiones (FontanA, 2008). Este tipo de vegetación presenta gran importancia ya que ha sido propuesta como indicador biológico de contaminación de aguas y de disturbios ambientales (MEIJER, 1976). FontanA (2014), menciona la desaparición de Wettsteiniola de los rápidos de Apipé, Ituzaingó (provincia de Corrientes) aguas abajo de la represa Yacyretá.

La mayoría de los estudios referidos a los invertebrados asociados a las plantas acuáticas en el nordeste de Argentina se realizaron en los humedales de grandes ríos como el Paraná (Poi DE NeIfF \& NeIFF, 2006). Los ensambles de invertebrados que habitan la vegetación reófila son muy poco conocidos $\mathrm{y}$, en algunos trabajos, se han identificado a los coleópteros acuáticos que polinizan a las plantas (THORP \& Rogers, 2015). En ríos de la región amazónica, SPRADA TAVAREZ et al. (2006) identificaron nueve especies de Podostemaceae y reconocieron 26 familias de insectos acuáticos o parcialmente acuáticos distribuidos en nueve órdenes. HAMADA (2000) destacó la importancia del estudio de los simúlidos relacionados con la vegetación reófila de diferentes ríos de Brasil. En Argentina, las referencias existentes para la provincia de Misiones tratan sobre los 
tricópteros (J. V. Sganga, datos inéditos) y la fauna de moluscos, peces, aves y mamíferos (CHATELLENAZ, 2007) asociados a la vegetación reófila. Además, un estudio preliminar realizado en el arroyo Urugua-í y tributarios, previo a la construcción de la represa que lleva el mismo nombre, analiza de manera cuali-cuantitativa la distribución espacio-temporal del bentos (PESO, 1995).

Podostemum ceratophyllum Michx. constituye diversos hábitats para la reproducción y la alimentación de los macroinvertebrados (Rosi-Marshall \& WaLlace, 2002) y sirve como sustrato para las algas epífitas que son la base de la red trófica en el río Tennessee.

Restos de hojas, tallos y semillas de Podostemaceae fueron encontrados en los estómagos de algunos peces de la región Amazónica (SANTOS et al., 1997; SANTOS \& RosA, 1998) y de la Selva Atlántica de Brasil (SilveIra Vilella et al., 2002), en pirañas del género Tometes (Guillaume, 2003) y en peces herbívoros del Alto Paraná. Estos hallazgos corroboran la importancia de las podostemáceas en las mallas tróficas de los ambientes reófilos.

El objetivo de este estudio fue (a) caracterizar el hábitat donde crecen Podostemum distichum (Cham.) Wedd. y Podostemum muelleri Warm. (Malpighiales: Podostemaceae) y (b) analizar la variación temporal de la abundancia y riqueza de taxa de los macroinvertebrados asociados a ambas especies que crecen en dos arroyos de bajo orden. Como hipótesis se postula que las plantas de Podostemaceae constituyen un hábitat propicio para los macroinvertebrados que varía según el sitio y la época del año.

\section{MATERIALES Y MÉTODOS}

Área de estudio. La provincia de Misiones tiene un clima subtropical húmedo sin estación seca y mesotermal, con veranos muy calurosos (promedios superiores a los $22{ }^{\circ} \mathrm{C}$ en el mes más cálido) e inviernos suaves. Las lluvias son intensas, con un promedio de 1900 a $2000 \mathrm{~mm}$ anuales (en años normales). De acuerdo a la clasificación de tipos climáticos de PaPADAKIs (1962), como consecuencia de la distribución de las lluvias a lo largo del año y a los excesos hídricos que se producen, el régimen pluviométrico de la provincia de Misiones es isohigro. Esto quiere decir que las estaciones hídricas son poco definidas y que en cualquier estación o mes, puede haber sequía o lluvias abundantes. Los milímetros de agua caídos durante el semestre frío tienden a igualar al semestre cálido (INTA, 2014).

El área de estudio se localiza en una cuenca que se caracteriza por tener arroyos que nacen en las sierras que atraviesan la provincia de Misiones, no superan los $70 \mathrm{~km}$ de extensión y desaguan en el río Uruguay. Se seleccionaron dos arroyos representativos: el arroyo Acaraguá $\left(27^{\circ} 16^{\prime} 17.6\right.$ ' S $\left.54^{\circ} 55^{\prime} 19.6^{\prime \prime} \mathrm{W}\right)$ que recibe sus aguas de los Cerros del Chapá y su afluente al arroyo del Medio $\left(27^{\circ} 14^{\prime} 9.3^{\prime \prime} \mathrm{S}-55^{\circ} 00^{\prime} 30.5^{\prime \prime} \mathrm{W}\right)$.

Metodología de muestreo. Para caracterizar el hábitat, en cada fecha de muestreo se registraron las variables físicas (profundidad) y químicas del agua $(\mathrm{pH}$, oxígeno disuelto y conductividad eléctrica), utilizando instrumentos digitales de medición. En laboratorio la concentración de nitrógeno total (nitritos + nitratos + amonio) y la de fósforo total fueron medidas por colorimetría (EATON et al., 1995). La concentración de clorofila $a$, fue medida por el método fluorométrico en muestras de agua de $500 \mathrm{ml}$ tomadas subsuperficialmente.

Durante el año 2011, se tomaron tres muestras de la especie de podostemácea dominante en cada arroyo en las cuatro estaciones del año, totalizando 24 muestras. A fin de recolectar los macroinvertebrados, se extrajo la vegetación reófila comprendida en un cuadrado de 40 x $40 \mathrm{~cm}\left(1600 \mathrm{~cm}^{2}\right)$ en cuyo extremo se colocó una red de dos $\mathrm{mm}$ de apertura de malla para evitar la pérdida del material por acción de la corriente del agua. Las muestras fueron colocadas en contenedores de cierre hermético y posteriormente fijadas con alcohol al 70\%. En el laboratorio, las plantas fueron lavadas cuidadosamente sobre recipientes de fondo blanco para facilitar la separación de los macroinvertebrados de la vegetación.

La identificación de las especies de podostemáceas se realizó siguiendo De Mello \& Fontana (2017) y la determinación taxonómica de los macroinvertebrados se realizó a nivel de familia siguiendo Domínguez \& FERNÁNDEZ (2009). El uso de la riqueza de familias, incluso de morfoespecies, ha sido aceptado en estudios realizados en ambientes tropicales y subtropicales (JACOBSEN et al., 2008), donde las descripciones de algunos taxones son aún incompletas y las claves taxonómicas específicas son escasas.

Las especies de Podostemaceae recolectadas fueron depositadas en el Herbarium Humboldtianum (CTESN) de la Facultad de Ciencias Exactas y Naturales y Agrimensura de la Universidad Nacional del Nordeste. Los macroinvertebrados estudiados se encuentran depositados en la colección del Centro de Ecología Aplicada del Litoral (CONICET-UNNE).

Análisis estadísticos. Para examinar el patrón espacial y temporal de los ensambles de macroinvertebrados, se ordenó la abundancia de las distintas familias utilizando un Análisis de Escalamiento Multidimensional No Métrico (NMDS). Los resultados fueron confirmados mediante un Análisis de Similaridades (ANOSIM) (Clarke, 1993), empleando la distancia de Bray-Curtis. A fin de comparar los resultados obtenidos en cada estación del año, se aplicaron test estadísticos de diversidad de familias $(\alpha)$ : índice de riqueza de familias (S) y los índices de MARGalef (1958) y MeNHINICK (1964). Las comparaciones de la riqueza y abundancia de las familias de macroinvertebrados entre arroyos y estaciones del año, se realizaron mediante análisis de la varianza no paramétrico de Kruskal-Wallis. La diversidad $\beta$ fue medida mediante los índices de similitud de Jaccard, de recambio de especies de Whittaker y de Complementariedad (CI). Los análisis estadísticos fueron realizados con el software PAST 2.08 (HAMmer, 2001).

\section{RESULTADOS}

Características del hábitat. Podostemum distichum fue la especie dominante en el arroyo Del Medio (sitio 1) y P. muelleri en el arroyo Acaraguá (sitio 2). A pesar de tratarse de especies distintas, las comunidades formadas por podostemáceas fueron muy similares en cuanto a su 
arquitectura y crecieron adheridas a las rocas formando una densa carpeta vegetal. Ambas especies presentaron una cobertura media del 100\% sobre el lecho de basalto. Podostemum distichum se desarrolló en aguas muy turbulentas y el estrato alcanzó $4 \mathrm{~cm}$ de altura, en cambio $P$. muelleri fue registrada en zonas con menor velocidad de la corriente y la altura del estrato fue menor $(3 \mathrm{~cm})$.

La profundidad del agua en el área ocupada por las podostemáceas varió entre 0.4 y $15 \mathrm{~cm}$. La máxima conductividad eléctrica $\left(40 \mu \mathrm{S} . \mathrm{cm}^{-1}\right)$ se registró en primavera y verano en ambos sitios y la menor $\left(20 \mu \mathrm{S} . \mathrm{cm}^{-1}\right)$ en el sitio 1 en otoño. El pH del agua varió entre 7.2 en primavera (sitio 1) y 7.8 ( sitio 2). La concentración promedio de oxígeno disuelto fue de $7.9 \mathrm{mg} / \mathrm{L}$ en el arroyo del Medio y de $8.6 \mathrm{mg} / \mathrm{L}$ en el arroyo Acaraguá. Con respecto a la composición iónica, el agua fue bicarbonatada magnésica-sódica en el sitio 1 y bicarbonatada magnésica-sódica-cálcica en el sitio 2 (Tab. I). En ambos arroyos el contenido de nutrientes (expresado por las diferentes formas de nitrógeno y el fosfato) fue bajo (Tab. I). Los valores de clorofila $a$ (Tab. I) indican que la biomasa de algas (fitoplancton) fue escasa en ambos arroyos.

Abundancia, riqueza de taxa y composición de los ensambles de macroinvertebrados. Se recolectó un total de 6675 macroinvertebrados en los dos sitios estudiados, y su abundancia total varió entre 312.5 y 6056.25 ind. $\mathrm{m}^{2}$ (Fig. 1). Las diferencias en la abundancia total no fueron significativas entre sitios (Kruskal-Wallis, $\mathrm{p}=0.386$ ), pero si entre las estaciones del año (Kruskal-Wallis, $\mathrm{p}=0.0051$ ).

Los ensambles de macroinvertebrados asociados a las podostemáceas estuvieron representadas por 14 familias en el sitio 1 y 13 familias en el sitio 2, distribuidas en nueve órdenes. La mayoría de ellas corresponden a

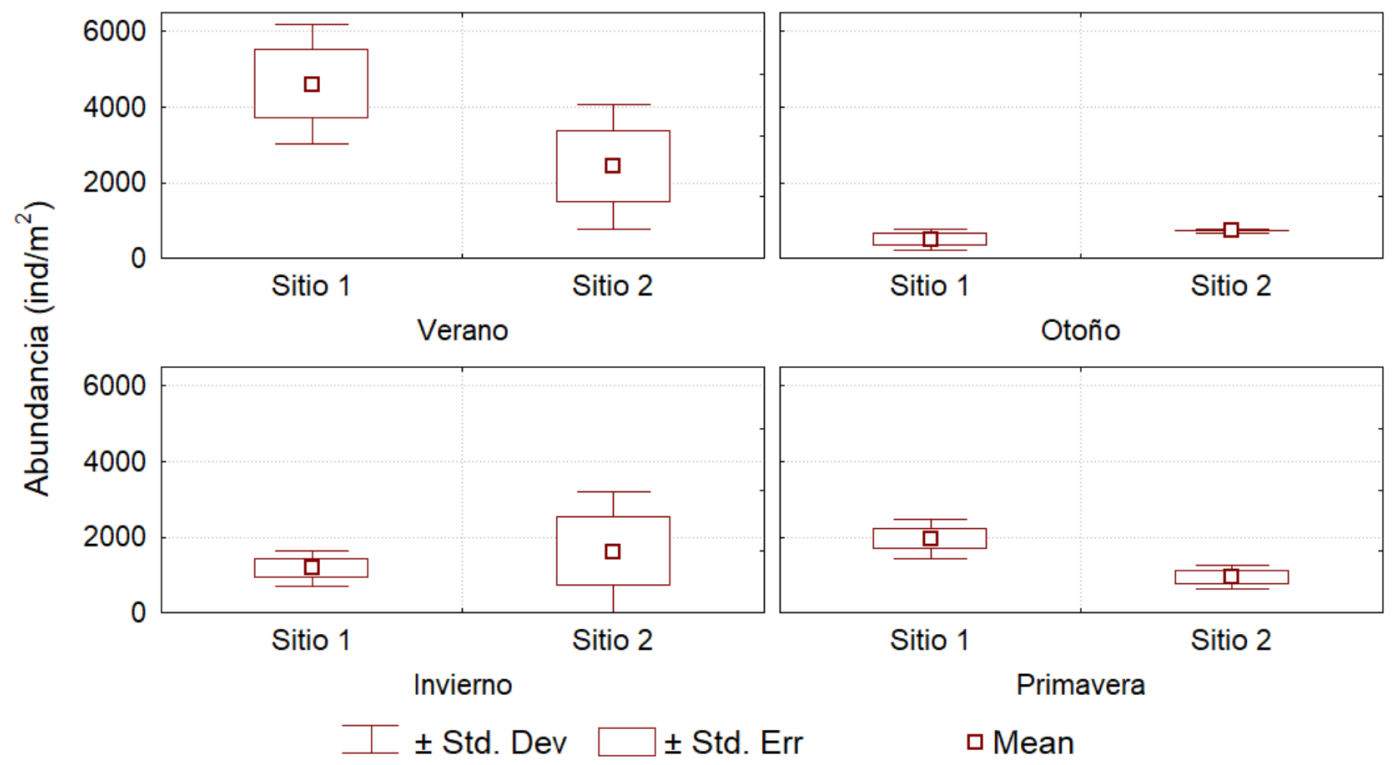

Fig. 1. Abundancia total de macroinvertebrados asociados a las podostemáceas en los sitios 1 y 2 durante las cuatro estaciones del año, Misiones, Argentina.

Tab. I. Principales características químicas de los sitios estudiados, Misiones, Argentina.

\begin{tabular}{lccc}
\hline & Unidad & Sitio 1 & Sitio 2 \\
\hline $\mathrm{pH}$ & Unidades & 5.28 & 4.92 \\
Conductividad & $\mu \mathrm{S} / \mathrm{cm}$ & 20 & 18 \\
Nitratos + Nitritos & $\mu \mathrm{g}\left(\mathrm{NO}_{2}^{-1}+\mathrm{NO}_{3}^{-1} / \mathrm{L}\right.$ & 20 & 15 \\
Amonio & $\mu \mathrm{g} / \mathrm{L}$ & 15 & 10 \\
Fosfatos & $\mu \mathrm{g} / \mathrm{L}$ & 10 & 10 \\
Magnesio & $\mathrm{mg} / \mathrm{L}$ & 1.5 & 1 \\
Potasio & $\mathrm{mg} / \mathrm{L}$ & 0.25 & 0.15 \\
Calcio & $\mathrm{mg} / \mathrm{L}$ & 0.5 & 1 \\
Sodio & $\mathrm{mg} / \mathrm{L}$ & 1 & 1 \\
Bicarbonatos & $\mathrm{mg} / \mathrm{L}$ & 10 & 7 \\
Sulfatos & $\mathrm{mg} / \mathrm{L}$ & 0.25 & \\
Cloruros & $\mathrm{mg} / \mathrm{L}$ & 1.0 & \\
D.Q.O (dis.) & $\mathrm{mg} \mathrm{O} / \mathrm{L}$ & 5.0 & \\
Clorofila $a$ & $\mu \mathrm{gg} / \mathrm{L}$ & $<5$ & 4.5 \\
\hline
\end{tabular}


estadios inmaduros y adultos de insectos con excepción de una familia de turbelarios (Planariidae) y de moluscos (Corbiculidae). Entre los insectos predominaron los estadios inmaduros de Diptera de dos familias (Chironomidae y Simuliidae), Ephemeroptera (Leptophlebiidae), Lepidoptera (Crambidae), Megaloptera (Corydalidae), Odonata (Libellulidae), Plecoptera (Gripopterygidae y Perlidae), Trichoptera (Hydrobiosidae e Hydropsychidae), y sólo en el orden Coleoptera (Elmidae y Gyrinidae) se encontraron, tanto estadios inmaduros como adultos.

De acuerdo al índice de Margalef el hábitat fue más diverso durante el otoño (3.37 y 3.15) en los sitios 1 y 2 , respectivamente. La menor diversidad estimada con el mismo índice fue 2.31 en primavera e invierno en ambos sitios.

La composición de las familias de macroinvertebrados fue muy similar en los sitios estudiados de acuerdo a los valores de diversidad beta medida con el índice de Jaccard (0.93) y el índice de complementariedad (0.07). El índice de Whittaker fue 0.27 , lo cual indica que el recambio de familias entre sitios fue bajo.

El Análisis de Escalamiento Multidimensional No Métrico (NMDS) de la abundancia de las distintas familias de macroinvertebrados, no mostró una clara separación entre ambos sitios de muestreo (Fig. 2). El análisis arrojó algunas diferencias en el patrón estacional (Fig. 2), segregando el otoño y el invierno del verano en ambos sitios (ANOSIM: $\mathrm{R}=0.29$; $\mathrm{p}=0.0001)$. El primer eje del análisis diferencia la abundancia de las distintas familias de macroinvertebrados en los distintos sitios, mientras que el segundo eje muestra similitudes entre las estaciones del año. Esta ordenación presentó un stress del 15\%.

La abundancia de las familias fue, en general, mayor durante el verano, excepto Hydrobiosidae e Hydropsychidae que resultaron ligeramente más numerosas en primavera en el sitio 1 (Fig. 3) e Hydrobiosidae con mayor número de individuos durante el invierno en el sitio 2 (Fig. 4). Las larvas de Simuliidae fueron abundantes en primavera y verano

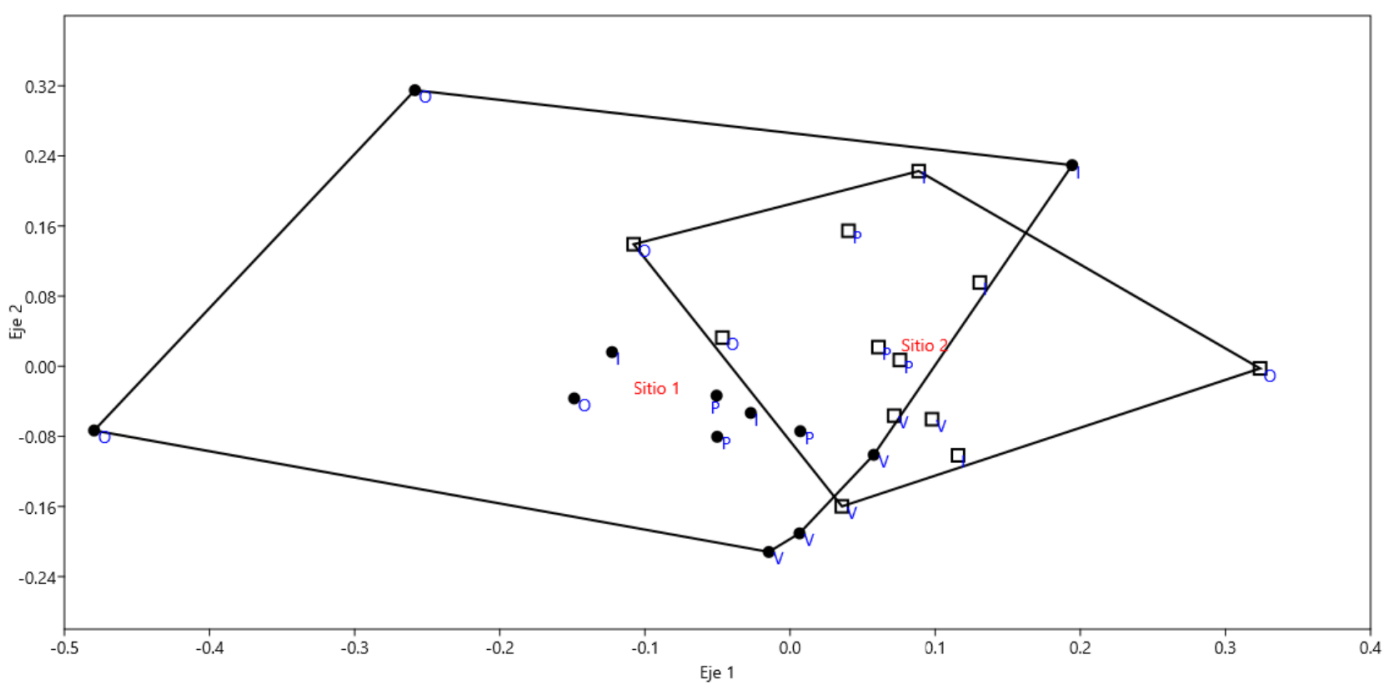

Fig. 2. NMDS. Abundancia de las familias de macroinvertebrados en los distintos sitios muestreados y estaciones del año (P, primavera; V, verano; O, otoño; I, invierno).

3

4
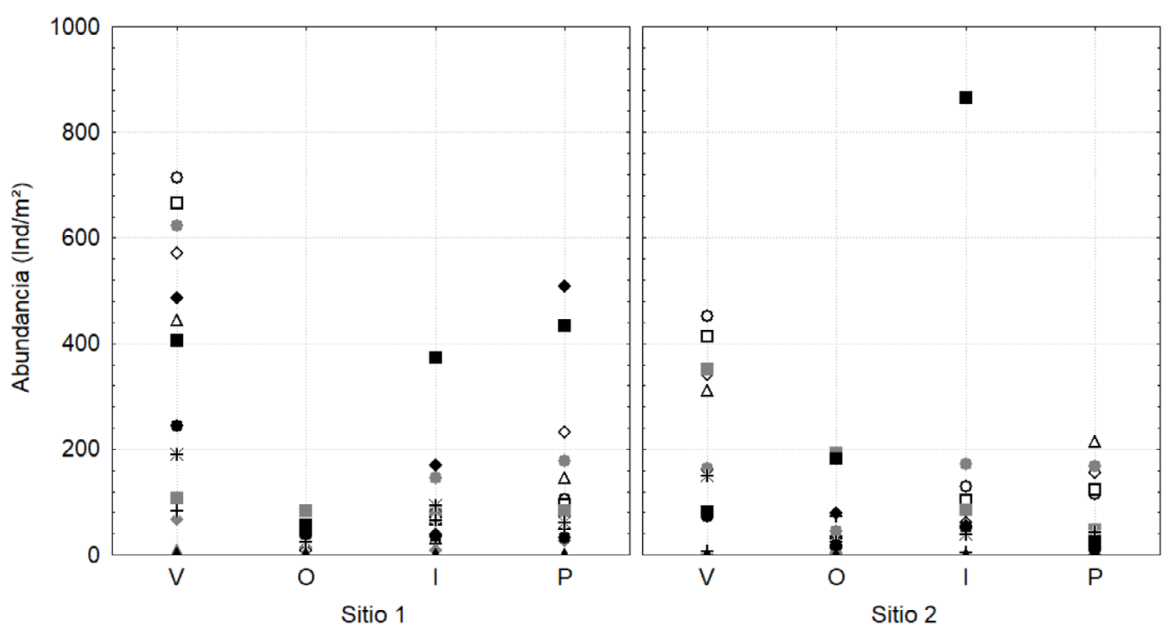

- GYR

口 ELM

$\diamond \mathrm{CHI}$

$\triangle \quad$ SIM

- LEP

- CRA

- COR

$\triangle \quad$ LIB

$+\quad$ PLA

* GRI

- Per

- hYD

- HYDR

$\Delta$ CORB

Figs 3, 4. Abundancia de las familias de macroinvertebrados hallados en los sitios estudiados en las distintas estaciones el año (V, verano; O, otoño; I, invierno; P, primavera). (GYR, Gyrinidae; ELM, Elmidae; CHI, Chironomidae; SIM, Simuliidae; LEP, Leptophlebiidae; CRA, Crambidae; COR, Corydalidae; LIB, Libellulidae; PLA, Planariidae; GRI, Gripopterygidae; PER, Perlidae; HYD, Hydrobiosidae; HYDR, Hydropsychidae; COR, Corbiculidae. 
y las diferencias entre estaciones fueron significativas en ambos sitios. En cambio, las larvas y adultos de Gyrinidae tuvieron diferencias significativas solo en el sitio 1 y las de Elmidae en el sitio 2. La abundancia de Leptophlebiidae (Ephemeroptera) e Hydropsychidae (Trichoptera) varió significativamente entre las estaciones del año en el sitio 1. Las larvas de Lepidoptera fueron abundantes durante el invierno con un pico poblacional de 291.66 individuos por $\mathrm{m}^{2}$.

\section{DISCUSIÓN}

Las características del hábitat (aguas cercanas al punto neutro, de baja salinidad y bicarbonatadas magnésicas - sódicas) son semejantes a la de otros tres arroyos de la misma cuenca (PoI DE NeIFF, 1999). En éstos, el fitoplancton es escaso (6-18 ind. $\mathrm{ml}^{-1}$ ) en coincidencia con la baja biomasa de algas encontrada en el presente estudio, lo que pone en relevancia la función de las Podostemaceae como productores primarios.

Los resultados indican que las dos especies de podostemáceas constituyen hábitats de diversidad intermedia de acuerdo al índice de diversidad utilizado. El número de órdenes de invertebrados registrado en los sitios estudiados fue similar al reportado para nueve especies de podostemáceas en arroyos de la región amazónica, pero la riqueza de familias fue menor (SPRADA TAVAREZ et al., 2006). En referencia a Trichoptera, otros rápidos con Podostemáceae de la provincia de Misiones fueron los hábitats más diversificados (J. V. Sganga, datos inéditos) con seis familias (Glossosomatidae, Hydropsychidae, Polycentropodidae, Sericostomatidae, Philopotamidae y Hydroptilidae). Para los Saltos del Moconá, Chatellenaz (2007) mencionó cuatro familias de moluscos asociados a las podostemáceas, de las cuales sólo Corbiculidae fue registrada en el presente estudio. Según el citado autor, las almejas del género Corbicula de origen asiático, ingresaron a nuestro país a comienzos de la década de 1970 y rápidamente se expandieron en los ríos que integran la Cuenca del Plata.

Los ensambles de macroinvertebrados del área de estudio tuvieron escasa variación espacial (bajo índice de reemplazo de especies), aun cuando en los arroyos estudiados dominaron diferentes especies de Podostemaceae. Este hecho se relaciona con la especialización del hábitat reófilo que requiere de adaptaciones a la vida en las aguas con alta velocidad de la corriente por parte de los invertebrados (HyNES, 1970).

Las diferencias estacionales fueron significativas al analizar la abundancia total y, según los resultados del NMDS, tuvieron un mayor efecto sobre la abundancia de las familias de macroinvertebrados. Distintas familias se alternaron en la dominancia numérica en las diferentes estaciones del año lo cual puede deberse a los ciclos estacionales de muda del estado de larva al de adulto. Por lo tanto, la hipótesis planteada es aceptada en parte. Si bien, la resolución taxonómica utilizada en este estudio permitió establecer algunas diferencias entre las estaciones del año, un nivel de resolución taxonómica menor podría revelar patrones más claros en el ordenamiento de los ensambles de invertebrados (KRATZER \& BATZER, 2007).

Las comunidades reófilas dominadas por Podostemaceae sustentan macroinvertebrados de diferentes funciones tróficas de acuerdo a la bibliografía disponible. Las familias más abundantes (Gyrinidae, Griopterigidae, Perlidae e Hydrobiosidae) fueron asignadas por MERRIT \& CUMMINS (1996) a la categoría trófica de depredadores. Según estos autores, otras familias procesan la materia orgánica particulada fina depositada (Elmidae y Leptophlebiidae) o en suspensión (larvas de Simuliidae) por lo cual pertenecen a la categoría trófica de colectores-recolectores y colectores-filtradores, respectivamente. Las larvas de lepidópteros (Crambidae) tuvieron una importante función como herbívoros dentro del hábitat estudiado.

Es necesario continuar con las investigaciones que comprendan otros cursos de agua del nordeste de Argentina lo que permitirá formular generalizaciones y profundizar las relaciones existentes entre estos singulares hábitat.

Agradecimientos. Parte de éste trabajo fue financiado por la Secretaría de Ciencia y Técnica de la Universidad Nacional del Nordeste, Argentina. PI Q001-2014. SGCYT (UNNE). Res. 984/14. Los autores agradecen al M.V. Egon Edvin Polej y al Dr. José Luis Fontana por su valiosa colaboración.

\section{REFERENCIAS}

Chambers, P. A.; Lacoul, P.; Murphy, K. J. \& Thomaz, S. M. 2008. Global diversity of aquatic macrophytes in freshwater. Hidrobiologia 595:9-26.

Chatellenaz, L. M. 2007. Fauna vinculada a la vegetación reófila del Parque Provincial Moconá Misiones, Argentina. FACENA 23:41-54.

Clarke, K. R. 1993. Non-parametric multivariate analysis of changes in community structure. Australian Journal of Ecology 18:117-143.

De Mello, A. S. \& Fontana, J. L. 2017. Familia Podostemaceae Rich. Ex Kunth. In: Anton, A. M.; Zuloaga, F. O. \& Belgrano, M. J. eds. Flora vascular de la República Argentina. Córdoba, Instituto Multidisciplinario de Biología Vegetal (CONICET-UNC), p. 315-323.

DomíngueZ, E. \& FernándeZ, H. R. 2009. Macroinvertebrados bentónicos sudamericanos Sistemática y biología. Tucumán, Fundación Miguel Lillo. 654p.

Eaton, A. D.; Clesceri, L. S. \& Greenberg, A. E. 1995. Standard Methods for the Examination of Water and Wastewater. 19ed. Washington, American Public Health Association (APHA). 1050 p.

Fontana, J. L. 2008. Alteraciones ambientales y comunidades de Podostemáceas en la represa de Yacyretá, Argentina y Paraguay. Available at $<$ http://www.unne.edu.ar/unnevieja/investigacion/ com2008/B-036.pdf $>$. Accessed on October 152018.

FontANA, J. L. 2014. La vegetación reófila del nordeste argentino. Las comunidades vegetales con Podostemaceae de la Provincia de Misiones. Boletín de la Sociedad Argentina de Botánica 49:115-136.

Guillaume, M. 2003. New herbivorous pirahnas discovered in french Guiana. Scientific Bulletins 168:1-2.

Hamada, N. 2000. Simulium (Psaroniocompsa) tergospinosum new Species (Diptera: Simuliidae) in siolii Group from the Southern Part of the State of Amazonas, Brazil. Anais do Instituto Oswaldo Cruz 95:819-828.

Hammer, Ø.; Harper, D. A. \& Ryan, P. D. 2001. PAST: Paleontological statistics software package for education and data analysis. Available at $<\mathrm{http}$ ://palaeo-electronica.org $>$. Accessed on October 182018.

HyNES, H. B. 1970. The ecology of running waters. Liverpool, Liverpool University Press. 555 p.

INTA. 2014. Instituto Nacional de Tecnología Agropecuaria (INTA). Available at $<$ http://inta.gob.ar/en/datoshistoricos $>$. Accessed on November 122018.

Jacobsen, D.; Cressa, C.; Mathooko, J. M. \& Dudgeon, D. 2008. Macroinvertebrates: composition, life histories and production. In: Dudgeon, D. ed. Tropical streams ecology. Amsterdam/Elsevier, Academic Press, p. 65-105.

Kratzer, E. B. \& Batzer, D. P. 2007. Spatial and temporal variation in aquatic macroinvertebrates in the Okefenokee swamp, Georgia, USA. Wetlands 27:127-140. 
Margalef, D. R. 1958. Information Theory in Ecology. General Systematics 3:36-71.

MeIJer, W. 1976. A note on Podostemum ceratophyllum Michx. as indicator of clean streams in and around the Appalachian Mountains. Castanea 41:319-324.

MenHINICK, E. F. 1964. A Comparison of some Species-Individuals Diversity Indices Applied to Samples of Field Insects. Ecology 45:859-861.

Merrit, R. W. \& Cummins, W. 1996. An introduction to the Aquatic Insects of North America. 3ed. Dubuque, Kendall/ Hunt. 862p.

NeIfF, J. J. 1986. Aquatic plants of the Paraná system. In: Davies, B. R. \& WalKer, K.F. eds. The Ecology of River Systems. Dr. W. Junk Publishers, Dordrecht, p. 557-571.

PAPADAKIS, J. 1962. Avances recientes en el estudio hídrico de los climas. Instituto de Suelos y Agrotecnia (Argentina) 175:1-28.

Peso, J. G. 1995. Zoobentos del arroyo Urugua-í. Estudio preliminar (Misiones, Argentina). Revista de la Asociación de Ciencias Naturales del Litoral 26:21-37.

PoI DE NeIFF, A. S. 1999. Capítulo IV.5. Aguas. In: Shell Capsa Forestal. Caracterización ambiental de los proyectos forestales de Shell Capsa. Informe final. Corrientes, Universidad Nacional del Nordeste, p. 319-362.

Poi de Neiff, A. S. \& NeIFF, J. J. 2006. Riqueza de especies y similaridad de los invertebrados que viven en plantas flotantes de la planicie de inundación del Río Paraná (Argentina). Interciencia 31:220-225.

Rosi-Marshall, E. J. \& Wallace , J. B. 2002. Invertebrate food webs along a stream resource gradient. Freshwater Biology 47:129-141.
SAnTos, G. M.; Pinto, S. S. \& Jegu, M. 1997. Alimentação do pacu-cana Mylesinus paraschomburgkii (Teleostei, Serrasalmidae) em rios da amazônia brasileira. Revista Brasileira de Biologia 57:311-315.

Santos, G. M. \& Rosa, P. S. 1998. Alimentação de Anostomus ternetzi e Synaptolaemus cingulatus, duas espécies de peixes amazônicos com boca superior. Revista Brasileira de Biologia 58:255-262.

Sprada Tavarez, A.; Odnetz, O. \& Enricone, A. 2006. A familia Podostemaceae em Rios Amazônicos e Comunidades de Insetos Asociados. Insula 35:19-50.

Thorp, J. H. \& Rogers, D. C. 2015. Ecology and General Biology. Thorp and Covich's Freshwater Invertebrates. 4ed. Amsterdam, Academic Press. 1118p.

TuR, N. M. 1975. Nueva especie de Podostemaceae para Argentina Wettsteiniola apipensis. Boletín de la Sociedad Argentina de Botánica 16:320-324.

Tur, N. M. 1997. Taxonomy of Podostemaceae in Argentina. Aquatic Botany 57:213-241. Special issue.

Tur, N. M. 1999. Podostemaceae. In: Sitchger, R. \& Ramella, L. Coord. Flora del Paraguay. Ginebra, Edit. des Cons. et Jard. Bot. de la ville de Genéve y Miss. Bot. Gard., p.1-35.

TuR, N. M. 2003. Una nueva especie de Marathrum (Podostemaceae) y nueva cita del género para la Argentina. Hickenia 3:151-198.

Vilella, F. S.; Becker, F. G. \& Hartz, S. M. 2002. Diet of Astyanax species (Teleostei, Characidae) in an Atlantic Forest River in Southern Brazil. Brazilian Archives of Biology and Technology 45:223-232. 